\title{
Controversial issues in radiotherapy for rectal cancer: a systematic review
}

\author{
Jong Hoon Kim, MD, PhD \\ Department of Radiation Oncology, Asan Medical Center, University of Ulsan College of Medicine, Seoul, Korea
}

The role of radiotherapy (RT) as an adjuvant to surgical options in the treatment of locally advanced rectal cancer has been established as it reduces local recurrence when combined with surgical resection and enhances survival when used in multidisciplinary treatment. However, many issues need to be addressed; some of these can render RT unnecessary, whereas others can reveal a new role of RT in rectal cancer. This review will discuss not only the basic role of RT but also the associated but controversial issues in detail in an attempt to find answers and determine future directions for the next decade.

Keywords: Rectal neoplasms, Radiotherapy, Margins of excision, Local excision

\section{Introduction}

The role of radiotherapy (RT) in the treatment of locally advanced rectal cancer (LARC) has been established in the last few decades. It is well known that RT can reduce local recurrence $(L R)$ when used combined with surgical resection and can enhance survival when used in multidisciplinary treatment (MDT). Although RT is accepted as an essential component of MDT, specific issues remain unaddressed. For example, it is necessary to define its role in specific subgroups, such as the intermediate-risk stage (T3NOMO) tumors or those involving the circumferential resection margin (CRM). $\mathrm{RT}$ is also associated with treatment-related toxicity that can be detrimental to the patient's quality of life (0OL). Without repeating textbook knowledge, this review, while briefly mentioning the basic facts of RT, will discuss controversial issues in detail to find answers and identify future directions for the next decade.

\section{Basic Role of Radiotherapy: Brief Review of Published Literature}

The first concern with RT as a local adjuvant treatment to surgical resection was whether it could reduce the local recurrence rate (LRR). In United States, patients underwent surgery and received postoperative RT, if needed, until 1990. In 1990, the National Cancer Institute Consensus Statement recommended the combined use of RT and 5-fluorouracil (5FU) for locally advanced ( $\mathrm{T} 3$ or $\mathrm{N}+$ ) rectal cancers [1]. The Consensus Statement was based on a few national randomized studies that showed a reduction in pelvic recurrence due to adjuvant RT and an increase in survival rates when combined with 5 -FU-based chemotherapy. The radiation dose used was approximately 45-50 Gy with 1.8-2 Gy fractions over 5 to 6 weeks; this is called conventional fractionation longcourse radiotherapy (LCRT). In contrast, in Europe, shortcourse radiotherapy (SCRT; 5 Gy $\times 5$ fractions/week) was used

Received 11 August 2017, Revised 28 September 2017, Accepted 02 October 2017.

Correspondence: Jong Hoon Kim, MD, PhD, Department of Radiation Oncology, Asan Medical Center, University of Ulsan College of Medicine, 88 Olympic-ro 43-gil, Songpa-gu, Seoul 05505, Korea. Tel: +82-2-3010-4434, Fax: +82-2-3010-6950, E-mail: jhkim2@amc. seoul.kr

(c) This is an Open Access article distributed under the terms of the Creative Commons Attribution Non-Commercial License (http://creativecommons.org/ licenses/by-nc/4.0/) which permits unrestricted non-commercial use, distribution, and reproduction in any medium, provided the original work is properly cited.

www.e-roj.org 
without chemotherapy before surgical resection. This type of preoperative RT was also effective in reducing LRR but did not improve survival rates, except in the Swedish Rectal Cancer Trial, although many meta-analyses reported a survival benefit [2-4].

The supportive role of RT was challenged with the introduction of total mesorectal excision (TME) because it significantly decreased LRR without adjuvant RT, thus questioning the necessity of RT before or after this new standard of surgery $[5,6]$. The Dutch Rectal Cancer Trial was the first to address and answer this question. In a randomized study that compared TME alone and preoperative SCRT plus TME, LRR was demonstrated to be significantly reduced in stage II and III rectal cancers with preoperative SCRT plus TME; an identical result was observed in the Stockholm TME project [7-9]. However, as toxicity and RT-associated complications remained a problem, it was thought necessary to avoid $\mathrm{RT}$ in patients with low risk of LR. Thus, the MRC group tried to reserve $\mathrm{RT}$ for patients with high risk factors that could be confirmed by surgical and pathological findings. They divided the patients into two groups where one group received preoperative RT and the other received TME alone. The TME arm also received selective postoperative chemoradiotherapy (CRT) if the pathology report was positive for CRM. Interestingly, patients could have been saved from unnecessary radiation exposure and medical expense if this trial had reported equivalent results. However, the results demonstrated superiority of preoperative RT in reducing LRR and increasing disease-free survival [10]. One interesting finding of this MRC study was that the difference in LRR was most prominent in the good-quality TME group.

\section{Should Radiotherapy Be Administered before or after Surgery?}

A few randomized studies have compared the effects of identical RT regimens delivered before and after surgery. A direct comparison between these two regimens using published literature is very difficult because fractionation schedules and interval-to-surgery are very different between the European and American studies and are affected by the prevailing socio-economic environment. Theoretically, preoperative RT has biological advantages as intact blood vessels and higher oxygenation status can contribute to higher radiosensitivity and the downsizing effect can be attributed to increased resectability and sphincter preservation rate. In contrast, the benefits of postoperative RT include selection of patients with high risk of $L R$ that can be verified by surgical and/or pathological findings. Nonetheless, advances in imaging techniques have made preoperative clinical staging more accurate, and patients with locally advanced tumors can benefit from preoperative RT. Three randomized studies have compared identical and concurrent CRT treatment before and after surgery [11-14]. However, two trials stopped earlier than initially planned because of low patient accrual rate. Thus, the German CAO/ARO/AIO 94 trial was the only randomized study with sufficient number of patients and definitive results. They enrolled 823 patients with cT3-4 or $\mathrm{N}+$ tumors with median follow-up period of nearly 4 years. The radiation dose was 50.4 Gy in conventional fractionation with two cycles of concurrent and continuous 5-FU infusion. They observed a lower LRR (13\% vs. $6 \% ; p=0.006$ ) with preoperative treatment but no significant improvement in disease-free survival (DFS) or overall survival (OS) rates; this effect persisted till 11 years of follow-up ( $7.1 \%$ vs. $10.1 \% ; p=0.048)$. Other benefits of preoperative treatment were higher sphincter preservation rate and lower acute and long-term complication rates. The two other randomized studies also reported favorable results for preoperative RT but differed in certain aspects from the German CAO/ARO/AIO 94 trial. The NSABP trial, which did not use TME as a standard surgical technique, reported higher DFS without any difference in LR and sphincter preservation rate, whereas the Korean study reported higher sphincter preservation rate with no difference in LR (Table 1) [11,12].

\section{Is SCRT alone better than LCRT?}

LCRT is expected to have the advantages of higher sphincter preservation rate as longer treatment period and interval-tosurgery will provide enough time for tumor shrinkage and downstaging effect. Further, complication rates will be lower as the higher number of fractionations will facilitate the successful repair of both normal tissue and organ damage. However, these advantages are associated with higher medical costs and more frequent visits to the hospital. To date, the two phase III randomized studies that have compared SCRT with LCRT are from Poland and Australia. Bujko et al. [15] compared $5 \times 5$ Gy SCRT with 50.4 Gy LCRT given in 28 fractions with 5-FU chemotherapy. They enrolled 200 clinical T3-4 middle or low rectal cancer patients but found no difference in oncological outcomes (local relapse-free survival, DFS, or OS at 4 years) despite higher pathological complete remission (pCR) rates and clear resection margins in the LCRT arm. Sphincter preservation rates were also similar between SCRT and LCRT, 
Table 1. Three randomized studies that have compared preoperative versus postoperative chemoradiotherapy

\begin{tabular}{|c|c|c|c|}
\hline & NSABP R-03 [11] & German $[13,14]$ & Korean [12] \\
\hline No. of patients & 267 & 799 & 240 \\
\hline CCRT regimen & $50.4 \mathrm{~Gy}+\mathrm{FL}$ & $50.4 \mathrm{~Gy}+5-\mathrm{FU} \mathrm{Cl}$ & 50 Gy + Cape. \\
\hline Location from AV (cm) & $\leq 15$ & $\leq 16$ & $\leq 10$ \\
\hline pCR $(\%)$ & 15 & 8 & 17 \\
\hline $5-y r$ LR (\%) & $\begin{array}{c}10.7 \text { vs } 10.7 \\
-\end{array}$ & $\begin{array}{c}6 \text { vs. } 13 \\
(p=0.006)\end{array}$ & $\begin{array}{c}5 \text { vs. } 6 \\
(p=0.335)\end{array}$ \\
\hline 5-yr DFS (\%) & $\begin{array}{c}64.7 \text { vs. } 53.4 \\
(p=0.011)\end{array}$ & $\begin{array}{l}68 \text { vs. } 65 \\
\text { (NS) }\end{array}$ & $\begin{array}{l}74 \text { vs. } 73 \\
(p=0.87)\end{array}$ \\
\hline $5-y r$ OS (\%) & $\begin{array}{c}74.5 \text { vs. } 65.6 \\
(p=0.065)\end{array}$ & $\begin{array}{c}76 \text { vs. } 74 \\
(p=0.8)\end{array}$ & $\begin{array}{l}85 \text { vs. } 83 \\
(p=0.62)\end{array}$ \\
\hline Spnincter preservation (\%) & $\begin{array}{c}47.8 \text { vs. } 39.2 \\
\quad(p=0.22)\end{array}$ & $\begin{array}{l}39 \text { vs. } 19 \\
(p=0.004)\end{array}$ & $\begin{array}{l}68 \text { vs. } 42 \\
(p=0.008)\end{array}$ \\
\hline Grade 3-5 acute toxicity (\%) & $\begin{array}{c}52 \text { vs. } 49 \\
-\end{array}$ & $\begin{array}{l}27 \text { vs. } 40 \\
(p=0.001)\end{array}$ & $\begin{array}{l}15 \text { vs. } 16 \\
(p=0.827)\end{array}$ \\
\hline
\end{tabular}

NSABP, National Surgical Adjuvant Breast and Bowel Project; CCRT, concurrent chemoradiation; FL, 5-fluorouracil and leucovorin; 5-FU $\mathrm{Cl}$, 5-fluorouracil continuous infusion; Cape., capecitabine; $A V$, anal verge; $p C R$, pathological complete response; $L R$, local recurrence; DFS, disease-free survival; OS, overall survival; NS, not significant.

Table 2. Comparison of short-course radiotherapy ( $5 \times 5$ Gy) and long-course chemoradiotherapy

\begin{tabular}{|c|c|c|}
\hline & Polish [15] & TROG [16] \\
\hline No. of patients & 312 & 326 \\
\hline CCRT regimen & $50.4 \mathrm{~Gy}+\mathrm{FL}$ & $50.4 \mathrm{~Gy}+5-\mathrm{FU} \mathrm{Cl}$ \\
\hline $\mathrm{RM}+(\%)$ & 12.9 vs. 4.4 & 5 vs. 4 \\
\hline \multirow[t]{2}{*}{$\mathrm{pCR}(\%)$} & 0.7 vs. 16.1 & - \\
\hline & $(p=0.017)$ & - \\
\hline \multirow[t]{2}{*}{ LR $(\%)$} & 10.6 vs. 15.6 (at 4 years) & 7.5 vs. 5.7 (at 5 years) \\
\hline & - & $(p=0.51)$ \\
\hline \multirow[t]{2}{*}{ DFS (\%) } & 58.4 vs. 55.6 (at 4 years) & - \\
\hline & - & $(p=0.47)$ \\
\hline \multirow[t]{2}{*}{ OS $(\%)$} & 67.2 vs. 66.2 (at 4 years) & 74 vs. 70 (at 5 years) \\
\hline & - & $(p=0.62)$ \\
\hline \multirow[t]{2}{*}{ Acute toxicity $\geq 3(\%)$} & 3.2 vs. 18.2 & - \\
\hline & $(p<0.001)$ & - \\
\hline \multirow[t]{2}{*}{ Late toxicity $\geq 3(\%)$} & 10.1 vs. 7.1 & - \\
\hline & - & $(p=0.53)$ \\
\hline Sphincter preservation (\%) & 47 vs. 42 & 63 vs. 69 \\
\hline
\end{tabular}

CCRT, concurrent chemoradiation; FL, 5-fluorouracil and leucovorin; 5-FU Cl, 5-fluorouracil continuous infusion; RM+, positive resection margin; pCR, pathological complete response; LR, local recurrence; DFS, disease-free survival; OS, overall survival.

which was unexpected. The authors explained that some surgeons had violated the study parameters by not preserving the anus even in pCR cases. Subsequently, Ngan et al. [16] reported similar results from the Trans-Tasman Radiation Oncology Group Trial where they enrolled 326 patients in a study design that was identical to that of the Polish trial, with the only difference being a trend of improved local control rate in distal rectal lesions. Therefore, they concluded that there was no difference in oncological outcomes or late toxicity (Table 2). Thus, there is no evidence to recommend one treatment modality over the other; the decision is made based on the patient's disease status and the physician's preference. If the tumor is located in the distal rectum and is close to the anal sphincter or is a locally advanced cT4 or CRM-positive lesion, LCRT would be chosen for sphincter preservation and complete resection of tumor, although enough supporting evidence is unavailable. 


\section{Should Adjuvant Radiotherapy $\mathrm{Be}$ Recommended in All Rectal Cancer Patients or Only in T3NO Cases of the Intermediate-Risk Group?}

Before answering this question, the pathological and clinical stages of the tumor should be distinguished. In cases of pathological T3NO tumors, the results of the older pooled analysis of data from three North American randomized studies showed that postoperative adjuvant RT may be excessive, as there was no visible effect of RT on local relapse, disease-free, or overall survival rates [17]. Further, TME, the current standard surgical technique results in much lower LRRs than those in the older studies. Although there are no randomized study results that support the omission of RT in this group, it appears that a subset of patients with pT3NO rectal cancer may have excellent outcomes with surgery alone or with surgery plus chemotherapy. Thus, it is reasonable to limit the use of RT to patients who show high risk factors, such as resection margin status, lymphovascular invasion, and poor differentiation, on pathological analysis.

Nonetheless, there has been a paradigm shift from postoperative to preoperative approach; in cases of clinically determined T3NOMO tumors, a very different situation is encountered. Recently, preoperative CRT has been widely adopted as the standard of care for LARC, and SCRT followed by immediate surgery is an alternative regimen preferred only in some European countries. The problem is that imaging studies have limitations in accurately staging cancers and can both over- and under-estimate disease status; it is not possible to know which one is predominant. However, the results of randomized studies show that the probability of understaging was more prevalent. In the Swedish Rectal Cancer Trial [2], LRR decreased from 21\% to 7\% in Dukes' stage B with preoperative $\mathrm{RT}$ alone, and survival rate also increased. In the Dutch trial that adopted TME as the standard surgical procedure, RT significantly lowered LRR (5.7\% vs. $1.0 \% ; p=0.01)$ in the stage II patient group, although it became non-significant at the 12year follow-up [8]. Similar findings were also observed in the MRC-CR07 study, which reported the effects of RT on local control in TNM stage II patients (6.4\% vs. $1.9 \%$ at 3 year; HR, $0.29 ; 95 \% \mathrm{Cl}, 0.12-0.67)[10]$.

Advances in imaging modalities are expected to result in greater accuracy in staging. MRI is one such modality that is expected to be of great value in accurate staging as it is can predict the need for complete resection with high specificity. A review of MRI-based staging in Europe by Smith and
Garcia-Aguilar [18] state that rectal cancers are stratified into different risk categories according to MRI findings (size, $\mathrm{T}$ - and $\mathrm{N}$-stage, location, extramural vascular invasion, and mesorectal fascia involvement) and that patients within each category are recommended appropriate treatment approaches as follows. Low-risk patients ( $L R R<10 \%$ ) are recommended TME alone, intermediate-risk patients (LRR 10\%-20\%) are advised preoperative SCRT followed by TME and adjuvant chemotherapy as standard treatment, and high-risk patients ( $L R R>20 \%$ ) are recommended preoperative CRT followed by TME and adjuvant chemotherapy. This strategy is quite different from the US guidelines and seems to be logical if MRI can be used as a reliable tool to differentiate among risk categories with high accuracy.

\section{Role of Radiotherapy in Incompletely Resected Cancers}

This is a very controversial issue among both radiation and surgical oncologists. Traditionally, RT was definitely administered before Marijnen's report [19], which refuted this concept. Subgroup analysis of the Dutch trial data revealed no difference in LRR between the preoperative SCRT and TME alone groups when CRM was $\leq 1 \mathrm{~mm}$, and postoperative RT did not affect LRR in CRM-positive lesions. If we follow their results, CRM-positive rectal cancers need not and should not be treated before or after surgical resection. Bujko and Kepka [20] pointed out a few critical defects in the Dutch study, such as insufficient patient numbers to draw clear conclusions, possibility of statistical fluctuation in subgroup analysis, and the role of RT proven by previous publications. Marijnen [21] agreed to most of the flaws raised by Bujko but did not change their conclusion and, instead, magnified the concerns raised in their reply to Bujko and Kepka [20]. They also insisted that LRR in irradiated patients with a positive CRM was 10 times higher than that in patients with a negative CRM. However, this is a very serious logical error as what they saw was the effect of CRM status in patients who received preoperative SCRT, and not the role of RT in CRM-positive patients. Although we can agree that CRM status is important in patients who receive RT, it does not mean that RT has no role in CRM-positive cases. Further, they compared LRR of CRM-positive and-negative groups ( $9.3 \%$ vs. $0.9 \%)$; if they had aimed to analyze whether RT can compensate for a positive resection margin, they should have compared LRR of CRM-positive patients who received RT (9.3\%) with that of clear/close margin patients who did not receive RT (14.9\% or $5.8 \%$ ). Such a comparison alone can 
help decide whether RT can compensate for CRM positivity. The average LRR in clear and close CRM cases is approximately $7 \%$, which is comparable to $9.3 \%$ observed in CRM-positive patients who received RT (Table 3 ).

Postoperative RT seems to have had no effect on LR in CRM-positive cases in the Dutch study. However, an analysis of patient characteristics showed that poor prognostic factors, such as number of APR cases and higher TNM stages, were more frequent in patients who received postoperative RT. Thus, their conclusions, based on simple comparisons should be interpreted very cautiously. Interestingly, another study that showed the importance of postoperative RT in CRMpositive patients was published 1 year later by the same Dutch group [22]. They reported that RT dose was an important factor that determined survival after $L R$, as among the many factors investigated, only RT dose was statistically significant ( $p=0.001$ ) and surgical resection of LR was only marginally significant ( $p=0.06$ ). Thus, Marijnen's conclusions [19], if true, do not concur with these results; it is very probable that RT is effective in CRM-positive cases with clinically measurable LR. Prospective randomized studies showed that preoperative SCRT can compensate for non-curative surgery. In the Swedish Rectal Cancer Trial, LRR in the RT plus surgery arm was 23\% in the non-curative surgery group, which is very similar to the $24 \%$ observed in the curative surgery alone arm [2]. In the non-curative surgery group, RT decreased LRR by $48 \%$ (44\% to $23 \%$ ); this reduction is very similar to the $43 \%$ decrease observed in the Dutch trial (16.4\% to 9.3\%) [7].

Another example is the MRC-CR07 study. They compared the 3-year LRR based on CRM involvement and between preoperative $\mathrm{RT}$ and selective postoperative CRT. LRR was $13.8 \%$ and $20.7 \%$ in the CRM-positive cases, indicating that preoperative $R T$ is more efficient in reducing $L R$ than selective postoperative treatment [10].

\section{Optimal Interval between Radiotherapy and Surgery in the Preoperative Approach Its Effects on Oncologic Outcomes}

Theoretically, a longer interval between the RT cessation and surgery can be translated as time sufficient for tumor shrinkage and downsizing. Thus, we can expect higher rates of pathological response and sphincter preservation. In fact, most studies that investigated the effect of interval time have reported an increase in the downsizing effect and higher pathological response rate, but no increase in sphincter preservation rates. Oncological outcomes, including LR and survival rates, were not different as radiation doses were constant in the study protocol. Another possible benefit of longer intervals is lower complication rates as the surrounding normal tissue has more time to recover from radiation-induced damage; however this effect was not clinically apparent. Nevertheless, greater fibrotic change in radiation field can make surgical resection more difficult and may also delay wound healing.

Lyon 90-01 was the first study that investigated the effect of the interval between RT and surgery (Table 4). They enrolled $>200$ patients with cT2-3 tumors that were amenable to digital rectal examination. A radiation dose of $39 \mathrm{~Gy}$ was given in 13 fractions, which is in between that of SCRT and LCRT. They divided the patients into short- (2 weeks) and long- (6-8 weeks) interval (SL and $\mathrm{LI}$, respectively) groups; the results were as expected. Specifically, the LI group showed higher rates of $p C R$ in the T-stage with prominent downsizing and downstaging effects. However, sphincter preservation rate did not improve in the distal rectal cancers even though the LI group showed a tendency toward better preservation (23\% vs. $41 \%$ ). LRRs were $9 \%$ in both groups; 3 -year survival rates showed no difference (SI group 78\% and LI group 71\%) [23].

Pach et al. [24] conducted another randomized study to assess the influence of time interval among patients who received preoperative SCRT ( $5 \times 5$ Gy). They enrolled 154 patients and randomly assigned them to 7-10 days

Table 3. CRM status and local recurrence in the Dutch trial [20]

\begin{tabular}{lccc}
\hline \multicolumn{1}{c}{ CRM status } & TME & RT + TME & p-value \\
\hline$>2 \mathrm{~mm}$ & $483(5.8)$ & $504(0.9)$ & $<0.001$ \\
$1-2 \mathrm{~mm}$ & $53(14.9)$ & $47(0)$ & 0.02 \\
$\leq 1 \mathrm{~mm}$ & $120(16.4)$ & $107(9.3)$ & 0.08 \\
Postoperative RT & $56(17.3)$ & - & - \\
No postoperative RT & $64(15.7)$ & - & \\
\hline
\end{tabular}

Values are presented as number (\% of local recurrence).

$\mathrm{CRM}$, circumferential resection margin; TME, total mesorectal resection; $\mathrm{RT}$, radiotherapy. 


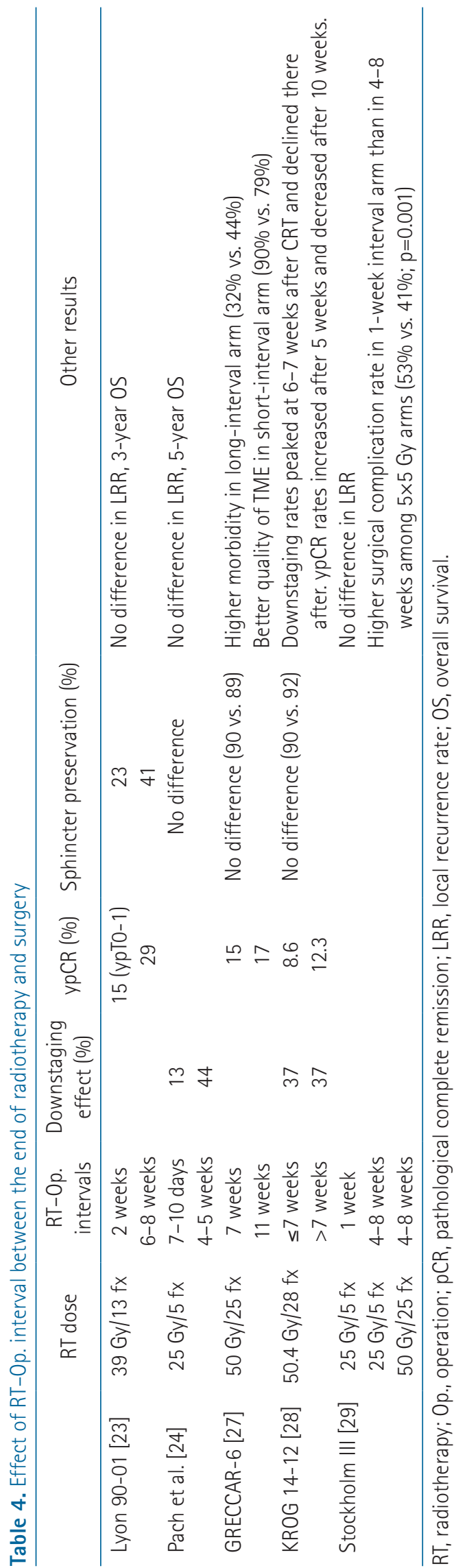

(SI) or 4-5 weeks (LI) subgroups. LI group patients showed higher downstaging rates than SI patients (44.2\% vs. 13\%; $p=0.0001)$, which was not associated with an increase in sphincter-saving procedures ( $p=0.627$ ) or curative resections $(p=0.132)$. No significant difference was observed in 5 -year survival (63\% and $73 \% ; p=0.24)$ or $L R(p=0.119)$ rates.

The absence of a sphincter-sparing effect in these randomized studies was predicted in the review article published by Bujko et al. [25] and was explained as follows: (1) irradiation does not meaningfully alter the distance between the anorectal ring and the lower pole of the lesion, (2) surgeons are not convinced by the suggestion or possibility of a shorter surgical distal bowel margin after irradiation $(<1 \mathrm{~cm}$ instead of the usually recommended 1-2 cm), and (3) cancer cells are found in the bowel wall in 40\%-75\% of postoperative specimens in cases of clinical CR. Gerard et al. [26], in a review article, have also reported no increase in sphincter preservation rate with LCRT.

GRECCAR- 6 was a multicenter randomized controlled study that compared 7- and 11-week intervals after neoadjuvant LCRT where the primary endpoint was $p C R$ rate defined as ypTONO [27]. pCR rate was not different among the two groups $(15.0 \%$ vs. $17.4 \%)$, but morbidity was significantly higher in the 11 -week group ( $44.5 \%$ vs. $32 \% ; p=0.0404$ ), and the 11 week group had a poorer quality of mesorectal resection (complete mesorectum, 78.7\% vs. 90\%; $p=0.0156$ ). However, sphincter preservation rates were similar in this study $(90.4 \%$ and $89.1 \%)$. Another large-scale multicenter study was reported from Korea was the KROG 14-12 study that assessed tumor response and aimed to identify the optimal operation time after preoperative CRT. They included data from 1,786 patients with LARC (cT3-4N0-2M0) who received preoperative CRT followed by TME and a radiation dose of $50.4 \mathrm{~Gy}$ in 28 fractions. Downstaging rates peaked between 6 and 7 weeks after CRT and declined thereafter, whereas ypCR rates increased from 5 weeks and decreased after 10 weeks. When they divided patients into early ( $\leq 7$ weeks) or delayed ( $>7$ weeks) surgery arms, the delayed arm tended to have higher sphincter preservation rates (92.4\% vs. 89.9\%; $p=0.078$ ) [28] Thus, from these studies, we can summarize that an increase in pathological response rate seems to reach its plateau around 6-7 weeks after LCRT but that no clinical advantage can be expected if interval time is $>10$ weeks and that longer intervals can make curative resection more difficult.

The recent Stockholm III trial focused on LRR based on interval time [29]. It was a multicenter, randomized, phase III non-inferiority study that compared $5 \times 5$ Gy with a 1-week interval-to-surgery, $5 \times 5$ Gy with a 4-8-week interval, and 25 
$\times 2$ Gy with 4- to 8-week interval. They enrolled 840 patients, with 385 patients in the three-arm randomization study and 455 in the two-arm randomization study that compared the two $5 \times 5$ Gy arms alone. As expected, there was no difference in LRR among the three arms. Interestingly, pooled analysis of the two SCRT regimens revealed that the incidence of postoperative complications was significantly lower after SCRT with delay than after SCRT with a short interval ( $p=0.001)$.

As summarized in Table 4, no oncological difference was found due to greater or lesser intervals between RT and surgery, but the rate of surgical complications were affected. In both SCRT and LCRT, a 4- to 8-week interval appears optimal for a higher response rate and reduced surgical complications compared with a 1-week interval for SCRT or 11-week interval for LCRT.

\section{Complications due to Radiotherapy}

It is well known that preoperative SCRT treatment increases surgical complication rates compared with surgery alone, as evidenced by many randomized trials. However, the type and severity of complications have been somewhat exaggerated and need to be clarified with a more objective interpretation of reported results. Marijnen et al. [30] cataloged acute complications of the Dutch TME trial and reported that RT toxicity rarely occurred but that greater blood loss during surgery and perineal complications in APR cases were the two statistically significant complications. In contrast, in a report on late complications, Peeters et al. [31] reported a significant decrease in patient satisfaction with respect to bowel function in the no-stoma group, a transient decrease in sexual activity in male patients until 6 months after LAR surgery, and decrease in sexual activity in female patients after 1 year. No difference was found in urinary or stoma function, but an age-matched control group for comparison was absent. Nonetheless, they concluded that although TME surgery was the main contributor of late bowel dysfunction rather than RT, fecal incontinence was more likely to occur after RT, especially if the perineum had been irradiated [32], but offered no explanation as to why perineal irradiation was important for incontinence. In another study and by cadaveric surgery, the authors clarified that the cause of the observed fecal and urinary incontinence was not RT but TME [33]. Specifically, they found that pelvic floor innervation can be damaged by low-lying tumors and that the origin of the levator ani nerve was located in close proximity to the origin of the pelvic splanchnic nerves. Thus, damage to pelvic floor innervation would contribute to fecal and urinary incontinence after TME, especially in cases of low anastomosis or damage to the pelvic splanchnic nerves. Subsequently, Lange et al. [32] reported that urinary dysfunction was not related to preoperative RT but to surgical nerve damage. Finally, in 2014, the Dutch group published their results of a 14-year follow-up on bowel and sexual function and concluded that there was no difference in overall functioning or health between TME alone and the preoperative RT plus TME groups [34].

Similar findings have also been reported by the MRC-CR07 study. The main adverse effect caused by surgery was sexual dysfunction; preoperative RT also led to sexual dysfunction and reduced bowel functioning [35].

Such thorough investigations have not been conducted for LCRT, but the results of the Stockholm III study showed that complication rates of LCRT were comparable to that of SCRT with a 4- to 8-week delay. However, SCRT followed by a 1 -week delay should be avoided as it is associated with much higher complication rates.

If we compare preoperative and postoperative CRTs in the German CAO/ARO/AIO 94 trial, grade 3 or 4 acute or late toxic effects were higher in the postoperative group than in the preoperative group (acute: $27 \%$ vs. 40\%, $p=0.001$; late: $14 \%$ vs. $24 \%, p=0.01$ ) [13]. However, in the Korean study, there was no difference in complication rates between the two groups, although the patterns of observed complications were slightly different [36]. Specifically, fistulous complications (anastomosis leakage and rectovaginal/rectovesical fistula, among others) were more frequent in the preoperative group, whereas obstructive complications (anastomotic stricture or small bowel obstruction) were more prevalent in the postoperative group.

\section{Organ Preservation with Radiotherapy}

\section{Wait-and-see in clinical complete responders} pCR (ypCR) rate after preoperative CCRT is reported to be approximately 15\%-20\% in phase III randomized trials $[11,13,37,38]$, and the prognosis in these patients is excellent. In a pooled analysis of data from 3,015 individual patients derived from 14 different datasets, ypCR patients showed very high 5-year survival (90\%) and DFS (87\%) rates [39]. In these pathological complete responders, radical resection may be an overtreatment that is usually accompanied by substantial morbidity and reduced $0 \mathrm{OL}$. However, it is not possible to predict pathological response before resection and clinical response does not indicate true pathological response with high accuracy. Habr-Gama et al. [40], the pioneer in organpreserving treatment, did not perform radical resection in 
clinical CR patients (watch-and-wait) and compared them to clinically PR but pathological CR cases that were established by analyzing surgical specimens. After a mean follow-up of 50 months, 5-year survival rates in clinical CR patients was $100 \%$ and DFS rate was 92\% with only three instances of LR among 71 patients. The study used an intensive followup protocol and performed resections if any tumor regrowth was observed. According to their long-term follow-up results, 183 patients with distal rectal cancer received neoadjuvant CCRT, and of these, 90 (49.2\%) did not need resection because of cCR [41]. Further, 62 of the 90 cCR patients were cured by non-surgical management, and 22 patients had LRs that could be successfully salvaged. Only six patients presented with nonresectable local failures. With this wait-and-watch strategy, 5 -year survival rate was $91 \%$ and DFS rate was $68 \%$, which are comparable to those of the cPR and resected groups (90\% and $87 \%$, respectively). Rectal preservation rate in the wait-andwatch group was 78\%, which is quite high for distal rectal cancers. These results are very optimistic but should only be carefully generalized as only six patients had shown excellent response to preoperative CRT and had been cured only when they received radical resection during $\mathrm{CCR}$. Important to note here is the cCR rate of nearly $50 \%$, which appears to be very high and not easily acceptable. Close follow-up is another issue that should be considered as it will increase medical expenditure and require patient access to cancer centers.

The Dutch investigators published a similar result but their ycCR rate was relatively lower than that in the study of Habr-Gama et al. [40] because the prospective cohort of 192 patients had T3-4 or N2 tumors [42]. Only 21 patients showed CCR (10.9\%), and there was only one LR that was salvaged by local excision (LE). Further, 10 of the 21 patients had distal rectal cancers and were deemed to have undergone the APR procedure. Smith et al. [43] retrospectively reviewed nonoperative management (NOM) and reports that of the 32 patients who were administered neoadjuvant CRT for stage I to III rectal cancer and were treated by NOM after cCR, only six demonstrated local failure. The control group, which received CRT followed by rectal resection and $\mathrm{pCR}$, had no instances of $L R$, and oncological outcomes were similar between the NOM and resection/pCR groups (2-year survival rates: 96\% vs. $100 \%, p=0.56$; distant DFS rates: $88 \%$ vs. $98 \%, p=0.27$ ). They concluded that rectal resection could be avoided in $81 \%$ of the patients selected for NOM and that it is possible to achieve degrees of local and distant disease control that are similar to what is observed in patients with pCR treated by rectal resection. However, as median follow-up periods were relatively short in these studies, it is necessary to wait for their long-term follow-up results as there may be additional recurrences. Importantly, we should remember that such excellent results with a wait-and-see policy were obtained under highly specific conditions and that there is insufficient evidence to accept this management regimen as standard treatment because no prospective randomized trial has been conducted to support such a recommendation.

\section{LE in good responders}

Another option for organ preservation in CCR patients is LE of the tumor site. The basic concept is that there could be a microscopic amount of residual tumor within radiation field and the most probable site would be the primary tumor bed because it has the highest tumor burden before treatment. If this highrisk tumor bed is removed by $L E$, patients can be cured with their rectum preserved and with less surgical morbidity. In addition it is possible to predict the pathological response of the lymph node if the pathological response of the rectal tumor is known. Kim et al. [44] reported that T-stage tumors were correlated to lymph node status after preoperative CRT, whereas the ypN stage was the strongest discrimination factor for DFS prediction. Therefore, information on ypT-stage after LE will help predict ypN stage and form the basis for determining the necessity for additional radical resection.

Before initiating this approach, it is necessary to decide the treatment endpoints based on tumor stage. For relatively early-stage cancers, such as CT2NO, the probability of pelvic lymph node metastasis is not high, and preoperative CRT is not recommended either before or after radical surgery. However, if the tumor is located in the distal rectum or if the patient is unfit for major surgery, preoperative CRT and LE represent a good alternative to TME that is without serious morbidity and does not require sacrificing the anus. In locally advanced cancers, such as T3 or node-positive tumors, this approach should be applied only in patients who can be categorized as good responders to preoperative CRT (ypT0-1). If ypT2-3 tumors are found in the pathological specimen after $L E$, radical dissection should be recommended as it is very probable that a malignant lymph node remains in the pelvis, and early studies have reported a high rate of locoregional recurrence. Nevertheless, early retrospective studies did not clearly define treatment endpoints according to tumor stage and treatment indicators may therefore be somewhat confusing. One such previous study by Lezoche et al. [45] investigated small T2-3 node-negative cases where they enrolled 100 patients and treated them with preoperative CRT followed by LE. 
Pathological stages observed were ypT0 $(n=3)$, microscopic residual $(n=15)$, ypT1 $(n=9)$, ypT2 $(n=54)$, and ypT3 $(n=19)$ tumors. Interestingly, they did not recommend major surgery in the ypT2-T3 cases. At a median follow-up of 55 months, LRR was $5 \%$ and distant metastasis occurred only in two patients. The cancer-specific survival rate at 90-month follow-up was $89 \%$, and the OS rate was 72\%, which seem unusually high on considering the large number of high ypT stages in the LE specimens. The small size of the tumors $(<3 \mathrm{~cm})$ in this study may be one possible explanation but is not enough to explain the observed results.

Stipa et al. [46] performed transrectal endoscopic microsurgery after preoperative CRT on 43 patients who had cT2-3 tumors. Of these, 17 patients had ypTO-1 with only one instance of $L R$, but in the 15 ypT2 patients, seven had LRs, five of whom had simultaneous distant failures. Among the 11 ypT3 patients, there were 9 LRs. Lee et al. [47] analyzed 27 cT3-4 patients who had ycCR following preoperative CRT but did not undergo radical surgery for various reasons. At a median follow-up of 81.8 months, 18 ypTo-1 patients showed 94.1\% local relapse-free survival (LRFS); the relapse-free and overall survival rates were $88.2 \%$ and $94.1 \%$. However, in ypT2-3 patients, LRFS was $77.8 \%$ and OS was $66.7 \%$. Borschitz et al. [48] reviewed data from 237 patients obtained from seven small studies and reported that LRR was $<5 \%$ in ypTO1 patients but $21 \%$ in ypT3 patients. Notably, the problems in these studies are their retrospective nature and the fact that data included patients with non-homogeneous stages.

Based on these results, it is possible to assume that ypT01 in LE specimen indicates high probability of local (and distal) control with a higher occurrence of ypNO [49]. Nonetheless, to be established as a reliable treatment, staging modalities should be standardized and it is necessary to define the patient group that will be benefitted most by this treatment using prospective studies.

ACOSOG Z6041 is the first multi-institutional prospective study that has investigated the oncological and functional outcomes of preoperative CRT and LE in patients with earlystage (T2NO) rectal cancers [50]. The eligibility criteria included clinical stage T2NO by EUS or endorectal coil MRI, $<4 \mathrm{~cm}$, $<40 \%$ of the circumference, and within $8 \mathrm{~cm}$ of the anal verge, among others. Treatment comprised capecitabine, oxaliplatin, and 54-Gy radiation, with the primary endpoint of 3-year DFS. At a median follow-up of 56 months, 3-year DFS was $88.2 \%$ in the intention-to-treat group and $86.9 \%$ in the per-protocol group. They concluded that preoperative CRT followed by LE might be considered as an organ-preserving alternative in CT2NO tumors, but there is insufficient evidence for this approach in locally advanced tumors that shows good response. Many retrospective studies have included various stages with distinct follow-up evaluations. Shaikh et al. [51] performed a systemic review and meta-analysis of oncological outcomes of LE and radical surgery after neoadjuvant CRT and concluded that there were no differences in LR, DFS and $0 S$ rates between the two groups despite cT3-4 stages being included in the analysis. It is not possible to draw any conclusions on whether LE can produce equivalent oncological outcomes in good responders to preoperative CRT for locally advanced tumors, but advances in imaging modalities may make such predictions possible if they can more accurately stage the tumors after CRT.

\section{Conclusion}

There is no doubt that RT is one of the essential components in the treatment of LARC, but its role has been challenged in certain situations, and RT-associated complications have made it a treatment modality that should be avoided if possible. However, such skepticism is being discounted by many investigators, and its use is being extended to earlier stages with the aim of organ preservation. In the next decade, we might see more radical changes in cancer treatment paradigms with RT.

\section{Conflict of Interest}

No potential conflict of interest relevant to this article was reported.

\section{References}

1. NIH consensus conference. Adjuvant therapy for patients with colon and rectal cancer. JAMA 1990;264:1444-50.

2. Swedish Rectal Cancer Trial, Cedermark B, Dahlberg M, et al. Improved survival with preoperative radiotherapy in resectable rectal cancer. N Engl J Med 1997;336:980-7.

3. Colorectal Cancer Collaborative Group. Adjuvant radiotherapy for rectal cancer: a systematic overview of 8,507 patients from 22 randomised trials. Lancet 2001;358:1291-304.

4. Camma C, Giunta M, Fiorica F, Pagliaro L, Craxi A, Cottone $M$. Preoperative radiotherapy for resectable rectal cancer: a meta-analysis. JAMA 2000;284:1008-15.

5. Heald RJ, Ryall RD. Recurrence and survival after total mesorectal excision for rectal cancer. Lancet 1986;1:1479-82.

6. MacFarlane JK, Ryall RD, Heald RJ. Mesorectal excision for 
rectal cancer. Lancet 1993;341:457-60.

7. Kapiteijn E, Marijnen CA, Nagtegaal ID, et al. Preoperative radiotherapy combined with total mesorectal excision for resectable rectal cancer. N Engl J Med 2001;345:638-46.

8. van Gijn W, Marijnen CA, Nagtegaal ID, et al. Preoperative radiotherapy combined with total mesorectal excision for resectable rectal cancer: 12-year follow-up of the multicentre, randomized controlled TME trial. Lancet Oncol 2011;12:575-82.

9. Martling $A L$, Holm T, Rutqvist LE, Moran BJ, Heald RJ, Cedemark B. Effect of a surgical training programme on outcome of rectal cancer in the County of Stockholm. Stockholm Colorectal Cancer Study Group, Basingstoke Bowel Cancer Research Project. Lancet 2000;356:93-6.

10. Sebag-Montefiore D, Stephens RJ, Steele R, et al. Preoperative radiotherapy versus selective postoperative in patients with rectal cancer (MRC CR07 and NCIC-CTG C016): a multicentre, randomised trial. Lancet 2009;373:811-20.

11. Roh MS, Colangelo LH, O'Connell MJ, et al. Preoperative multimodality therapy improves disease-free survival in patients with carcinoma of the rectum: NSABP R-03. J Clin Oncol 2009;27:5124-30.

12. Park JH, Yoon SM, Yu CS, Kim JH, Kim TW, Kim JC. Randomized phase 3 trial comparing preoperative and postoperative chemoradiotherapy with capecitabine for locally advanced rectal cancer. Cancer 2011;117:3703-12.

13. Sauer $R$, Becker $H$, Hohenberger W, et al. Preoperative versus postoperative chemoradiotherapy for rectal cancer. N Engl J Med 2004;351:1731-40.

14. Sauer R, Liersch T, Merkel $S$, et al. Preoperative versus postoperative chemoradiotherapy for locally advanced rectal cancer: results of the German CAO/ARO/AIO-94 randomized phase III trial after a median follow-up of 11 years. J Clin Oncol 2012;30:1926-33.

15. Bujko K, Nowacki MP, Nasierowska-Guttmejer A, Michalski W, Bebenek $M$, Kryj M. Long-term results of a randomized trial comparing preoperative short-course radiotherapy with preoperative conventionally fractionated chemoradiation for rectal cancer. Br J Surg 2006;93:1215-23.

16. Ngan SY, Burmeister B, Fisher RJ, et al. Randomized trial of short-course radiotherapy versus long-course chemoradiation comparing rates of local recurrence in patients with $\mathrm{T} 3$ rectal cancer: Trans-Tasman Radiation Oncology Group trial 01.04. J Clin Oncol 2012;30:3827-33.

17. Gunderson LL, Sargent DJ, Tepper JE, et al. Impact of T and N stage and treatment on survival and relapse in adjuvant rectal cancer: a pooled analysis. J Clin Oncol 2004;22:1785-96.

18. Smith JJ, Garcia-Aguilar J. Advances and challenges in treatment of locally advanced rectal cancer. J Clin Oncol 2015:33:1797-808.

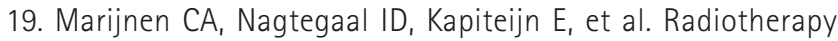
does not compensate for positive resection margins in rectal cancer patients: report of a multicenter randomized trial. Int J Radiat Oncol Biol Phys 2003;55:1311-20.

20. Bujko K, Kepka L. In regard to Marijnen et al.: does radiotherapy compensate for a positive resection margin in rectal cancer patients? IJROBP 2003;55:1311-1320. Int J Radiat Oncol Biol Phys 2003;57:1199-200.

21. Marijnen C. In response to Drs. Bujko and Kepka. Int J Radiat Oncol Biol Phys 2003;55;1200.

22. van den Brink $M$, Stiggelbout $A M$, van den Hout WB, et al. Clinical nature and prognosis of locally recurrent rectal cancer after total mesorectal excision with or without preoperative radiotherapy. J Clin Oncol 2004;22:3958-64.

23. Francois Y, Nemoz CJ, Baulieux J, et al. Influence of the interval between preoperative radiation therapy and surgery on downstaging and on the rate of sphincter-sparing surgery for rectal cancer: the Lyon R90-01 randomized trial. J Clin Oncol 1999;17:2396.

24. Pach R, Kulig J, Richter P, Gach T, Szura M, Kowalska T. Randomized clinical trial on preoperative radiotherapy 25 Gy in rectal cancer--treatment results at 5-year follow-up. Langenbecks Arch Surg 2012;397:801-7.

25. Bujko K, Kepka L, Michalski W, Nowacki MP. Does rectal cancer shrinkage induced by preoperative radio(chemo)therapy increase the likelihood of anterior resection? A systematic review of randomised trials. Radiother Oncol 2006;80:4-12.

26. Gerard JP, Rostom Y, Gal J, et al. Can we increase the chance of sphincter saving surgery in rectal cancer with neoadjuvant treatments: lessons from a systematic review of recent randomized trials. Crit Rev Oncol Hematol 2012;81:21-8.

27. Lefevre JH, Mineur L, Kotti S, et al. Effect of Interval (7 or 11 weeks) Between Neoadjuvant Radiochemotherapy and Surgery on Complete Pathologic Response in Rectal Cancer: A Multicenter, Randomized, Controlled Trial (GRECCAR-6). J Clin Oncol 2016 Jul 18 [Epub]. http://doi.org/10.1200/ JC0.2016.67.6049.

28. Kwak YK, Kim K, Lee JH, et al. Timely tumor response analysis after preoperative chemoradiotherapy and curative surgery in locally advanced rectal cancer: a multi-institutional study for optimal surgical timing in rectal cancer. Radiother Oncol 2016;119:512-8.

29. Erlandsson J, Holm T, Pettersson D, et al. Optimal fractionation of preoperative radiotherapy and timing to surgery for rectal cancer (Stockholm III): a multicentre, randomised, 
non-blinded, phase 3, non-inferiority trial. Lancet Oncol 2017;18:336-46.

30. Marijnen CA, Kapiteijn E, van de Velde CJ, et al. Acute side effects and complications after short-term preoperative radiotherapy combined with total mesorectal excision in primary rectal cancer: report of a multicenter randomized trial. J Clin Oncol 2002;20:817-25.

31. Peeters KC, van de Velde CJ, Leer JW, et al. Late side effects of short-course preoperative radiotherapy combined with total mesorectal excision for rectal cancer: increased bowel dysfunction in irradiated patients: a Dutch colorectal cancer group study. J Clin Oncol 2005;23:6199-206.

32. Lange $M M$, den Dulk $M$, Bossema ER, et al. Risk factors for faecal incontinence after rectal cancer treatment. Br J Surg 2007:94:1278-84.

33. Wallner $C$, Lange MM, Bonsing BA, et al. Causes of fecal and urinary incontinence after total mesorectal excision for rectal cancer based on cadaveric surgery: a study from the Cooperative Clinical Investigators of the Dutch total mesorectal excision trial. J Clin Oncol 2008;26:4466-72.

34. Wiltink LM, Chen TY, Nout RA, et al. Health-related quality of life 14 years after preoperative short-term radiotherapy and total mesorectal excision for rectal cancer: report of a multicenter randomised trial. Eur J Cancer 2014;50:2390-8.

35. Stephens RJ, Thompson LC, Quirke P, et al. Impact of shortcourse preoperative radiotherapy for rectal cancer on patients' quality of life: data from the Medical Research Council CR07/ National Cancer Institute of Canada Clinical Trials Group C016 randomized clinical trial. J Clin Oncol 2010;28:4233-9.

36. Kim CW, Kim JH, Yu CS, et al. Complications after sphinctersaving resection in rectal cancer patients according to whether chemoradiotherapy is performed before or after surgery. Int J Radiat Oncol Biol Phys 2010;78:156-63.

37. O'Connell MJ, Colangelo LH, Beart RW, et al. Capecitabine and oxaliplatin in the preoperative multimodality treatment of rectal cancer: surgical end points from National Surgical Adjuvant Breast and Bowel Project trial R-04. J Clin Oncol 2014;32:1927-34.

38. Bosset JF, Calais G, Mineur L, et al. Enhanced tumorocidal effect of chemotherapy with preoperative radiotherapy for rectal cancer: preliminary results: EORTC 22921. J Clin Oncol 2005;23:5620-7.

39. Maas M, Nelemans PJ, Valentini $V$, et al. Long-term outcome in patients with a pathological complete response after chemoradiation for rectal cancer: a pooled analysis of individual patient data. Lancet Oncol 2010;11:835-44.
40. Habr-Gama A, Perez RO, Nadalin W, et al. Operative versus nonoperative treatment for stage 0 distal rectal cancer following chemoradiation therapy: long-term results. Ann Surg 2004;240:711-8.

41. Habr-Gama A, Gama-Rodrigues J, Sao Juliao GP, et al. Local recurrence after complete clinical response and watch and wait in rectal cancer after neoadjuvant chemoradiation: impact of salvage therapy on local disease control. Int J Radiat Oncol Biol Phys 2014;88:822-8.

42. Maas M, Beets-Tan RG, Lambregts DM, et al. Wait-and-see policy for clinical complete responders after chemoradiation for rectal cancer. J Clin Oncol 2011;29:4633-40.

43. Smith JD, Ruby JA, Goodman KA, et al. Nonoperative management of rectal cancer with complete clinical response after neoadjuvant therapy. Ann Surg 2012;256:965-72.

44. Kim DW, Kim DY, Kim TH, et al. Is T classification still correlated with lymph node status after preoperative chemoradiotherapy for rectal cancer? Cancer 2006;106:1694-700.

45. Lezoche E, Guerrieri M, Paganini AM, Baldarelli M, De Sanctis A, Lezoche $G$. Long-term results in patients with T2-3 No distal rectal cancer undergoing radiotherapy before transanal endoscopic microsurgery. Br J Surg 2005;92:1546-52.

46. Stipa F, Picchio M, Burza A, Soricelli E, Vitelli CE. Long-term outcome of local excision after preoperative chemoradiation for ypT0 rectal cancer. Dis Colon Rectum 2014;57:1245-52.

47. Lee NK, Kim DY, Kim SY, et al. Clinical outcomes of local excision following preoperative chemoradiotherapy for locally advanced rectal cancer. Cancer Res Treat 2014;46:158-64.

48. Borschitz $T$, Wachtlin $D$, Mohler $M$, Schmidberger $H_{\text {, Junginger }}$ T. Neoadjuvant chemoradiation and local excision for T2-3 rectal cancer. Ann Surg Oncol 2008;15:712-20.

49. Yeo SG, Kim DY, Kim TH, et al. Pathologic complete response of primary tumor following preoperative chemoradiotherapy for locally advanced rectal cancer: long-term outcomes and prognostic significance of pathologic nodal status (KROG 0901). Ann Surg 2010;252:998-1004.

50. Garcia-Aguilar J, Renfro LA, Chow OS, et al. Organ preservation for clinical T2NO distal rectal cancer using neoadjuvant chemoradiotherapy and local excision (ACOSOG Z6041): results of an open-label, single-arm, multi-institutional, phase 2 trial. Lancet Oncol 2015;16:1537-46.

51. Shaikh I, Askari A, Ourû S, Warusavitarne J, Athanasiou T, Faiz 0. Oncological outcomes of local excision compared with radical surgery after neoadjuvant chemoradiotherapy for rectal cancer: a systematic review and meta-analysis. Int J Colorectal Dis 2015;30:19-29. 